\title{
Understanding 'How':
}

Two kinds of mechanistic explanations underlie known explanation preferences

Sehrang Joo, Sami R. Yousif, and Frank C. Keil

Department of Psychology, Yale University

\begin{tabular}{|c|c|}
\hline Running head & Understanding 'How' \\
\hline Institution address & $\begin{array}{l}2 \text { Hillhouse Avenue } \\
\text { Yale University } \\
\text { New Haven, CT, USA } 06511\end{array}$ \\
\hline Email addresses & sehrang.joo@yale.edu \\
\hline Version & Preprint 04/21/21 \\
\hline
\end{tabular}




\begin{abstract}
Parallel research programs across decades have developed contrasting accounts of people's explanation preferences. One perspective emphasizes adults' and children's preferences for teleological explanations (i.e., explanations referring to something's purpose), even in direct contrast with mechanistic (or causal) explanations. The other perspective highlights contexts where people instead seek out mechanistic knowledge and judge it to be particularly valuable. These characterizations of people's explanation preferences support fundamentally different theories of people's intrinsic worldviews: People may either be irrational and prone to unscientific explanation, or relatively sophisticated investigators of the world around them. How can these teleo-centric and mech-centric views of explanation preferences be reconciled? Here, we demonstrate that mechanistic explanations are comprised of two significant subtypes of explanation. Etiological mechanisms address how things came to be, whereas constitutive mechanisms address how they currently work. In Experiments 1 and 2, we find that people prefer constitutive mechanisms to etiological mechanisms. In Experiments 3, 4a, and 4b, we also find that constitutive and etiological mechanisms are judged differently against teleological explanations. In general, constitutive mechanisms perform better against teleological explanations than etiological mechanisms. Thus, people's preferences depend on the type of mechanism involved; they may prefer teleology to one kind of mechanism but not to the other. We discuss implications for the larger debate on explanation preferences.
\end{abstract}




\section{Introduction}

Explanations can profoundly shape how we understand the world. As such, our preferences for certain types explanation are critical to accounts of how this understanding develops. Two major views in cognitive science diverge on this question. On one view, people both seek and prefer mechanistic explanations, or explanations that capture the rich causal structure underlying an object or event (e.g., Callanan \& Oakes, 1992; Greif et al., 2006; Trouche et al., 2018). For example, we might explain that "A clock ticks because an internal motor turns a gear which moves the hands" or "The Grand Canyon came to exist because the river caused erosion of the rock over time." On the alternative view, people instead privilege teleological explanations, or explanations that appeal to something's purpose (e.g., Kelemen, 1999; Kelemen et al., 2013; Heywood \& Bering, 2014). According to this view, people prefer explanations such as "The clock ticks so that it can tell time" or "The Grand Canyon exists to provide a beautiful and unique ecosystem," even over and above causal explanations.

More than mere descriptions of explanation preferences, these 'mech-centric' and 'teleocentric' views offer distinct, even opposite, accounts of human cognition (e.g., Keil \& Lockhart, in press; Kelemen, 2004)—yet both views are supported by well-developed research programs spanning multiple decades (e.g., Chuey et al., 2020; Gelman \& Wellman, 1991; Kelemen, 1999; Rose, forthcoming). On the mech-centric view, adults and children are fundamentally curious and seek out the right kinds of information to make sense of our rich causal world. In stark contrast, the strong interpretation of the teleo-centric view holds that humans are intrinsically unscientific and that children are natively theist. How, then, can these views be reconciled? Here, we suggest one way of resolving these competing bodies of work. We propose that the contrast 
between mechanistic and teleological explanations is an oversimplified dichotomy, one that fails to account for relevant sub-types of explanations.

\section{The mech-centric view of explanation}

Classic work demonstrates that even children have a straightforward preference for mechanistic explanation. They prefer causal explanations to mere statements of fact (Corriveau \& Kurkul, 2014; Frazier et al., 2009; Frazier et al., 2016) and seek out even non-obvious causal information about entities' internal properties (German \& Wellman, 1991). When asking openended questions, they ask teleological questions only when adults would also find such questions reasonable (e.g., about parts of animals but not animals themselves; Greif et al., 2006) and otherwise ask an increasing number of causal "how" or "why" questions throughout early childhood (Callanan \& Oakes, 1992; Chouinard, 2007; Engel, 2011). As young as five years old, children will specifically choose to learn about mechanism, choosing a book that "tells you about how the parts work together to make it do things" over one offering teleological or merely factual information (Trouche et al., 2018), and think that someone with mechanistic knowledge knows more than someone with different, equally complex, knowledge of other explanations (Lockhart et al., 2019).

On this mech-centric view, people's explanation preferences feed naturally into how we learn and discover scientifically relevant information from a young age. While even adults may not regularly possess deep mechanistic knowledge of everyday phenomenon (see Rozenblit \& Keil, 2002), people nevertheless recognize the value of such information and regularly seek it out. They recognize that mechanistic information generalizes to superordinate categories (e.g., someone who knows mechanistic information about clocks or sparrows also knows about machines or birds; Chuey et al., 2020), and that mechanisms often shape natural categories 
(Erickson et al., 2010) as well as types of scientific inquiry (Keil et al., 2008; Keil et al., 2010). These inferences support rich, scientifically valid learning. An interest in mechanism not only suggests that children's inability to verbally differentiate between causal patterns "should not be confused with problems in understanding such differences" (Grief et al., 2006, p.459), but also that "thinking of the world in mechanistic terms is both cognitively natural and motivating for humans" (Keil \& Lockhart, in press).

\section{The teleo-centric view of explanation}

Other work demonstrates a preference for teleological explanations. Children, and even adults, seem to be 'promiscuously' teleological—preferring teleological explanations of a wide variety of phenomenon, including non-living natural kinds like trees or the sun. When faced with an ambiguous 'why' question about an animal or artifact, adults often choose teleological explanations over mechanistic explanations (Kelemen, 1999). Under speeded conditions, adults also endorse teleological explanations more broadly, agreeing with statements such as "Rain falls in order to allow plants to grow" (Kelemen et al., 2013; Kelemen \& Rosset, 2009). In general, people tend to attribute teleology to a wide variety of things, including natural kinds (Heywood \& Bering, 2014; see also Kelemen et al., 2013; Kelemen \& Rosset, 2009), life events (Banerjee \& Bloom, 2015), and even human beings (Lewry et al., 2020). These tendencies are exacerbated in children, who tend to choose teleological explanations over mechanistic ones across domains (Banerjee \& Bloom, 2015; Kelemen, 1999; Kelemen \& DiYanni, 2009) and across cultures (Schachner et al., 2016).

Yet teleological explanations are properly causal explanations only in cases where the explanandum's purpose actually led to its existence. In other words, whereas teleology may provide an appropriate causal history for intentionally designed artifacts (e.g., a knife is actually 
sharp because it was designed for that purpose), teleological explanations about other sorts of entities present faulty causal stories (Kelemen, 2004; Kelemen \& Rosset, 2009; Kelemen et al., 2013). To say that the Grand Canyon exists so that it can provide an ecosystem, for instance, suggests that it was intentionally designed for this purpose. People's tendency to imbue much of the world with purpose is therefore taken as evidence that children in particular are intuitive theists (Kelemen, 2004), and that this default worldview continues to color how we instinctively see the world through adulthood (Kelemen \& Rosset, 2009; Kelemen et al., 2013; Rose, 2015; Rose \& Schaffer, 2017; Rose et al., 2020; Rose, forthcoming). In other words, the teleo-centric view supports the theory that "we view nature as a whole as being infused with agency" (Rose, forthcoming, p. 12). Indeed, this default cognitive bias may "have subtle enduring effects on our species' intellectual progress, creating impediments for truly mechanistic understanding and discovery” (Kelemen et al., 2013, p. 1081).

\section{Resolving the debate: Two kinds of mechanism?}

These perspectives are fundamentally at odds. Despite decades of relevant research, no proposal has offered a clear way to understand how both of these sets of results might cohere. Here, we offer one possible way to understand and potentially resolve these differences. We propose that the contrast between teleological and mechanistic explanations neglects critical contrasts between major explanatory kinds. Mechanistic explanations are comprised of two qualitatively different subtypes — etiological vs. constitutive - that have been confounded in prior work.

For nearly as long as psychologists have investigated mechanistic vs. teleological explanations, philosophers of science have distinguished between these subtypes of mechanism (Salmon, 1984). Etiological mechanisms describe a chain of events that cause a certain 
phenomenon, or, in other words, how things came to be. An account of how a tree grew its leaves, for instance, is an etiological mechanistic explanation (see also Skipper and Milstein, 2004; Barros, 2008, Glennan, 2009 for examples of etiological mechanisms). Constitutive mechanisms, in contrast, describe an ongoing causal process by reference to the interaction of smaller parts: They explain how things work. Take, for example, an explanation of how a clock ticks: This explanation would refer to the internal makeup of the clock and describes how different parts move and work together to create the phenomenon in question (see also Bechtel and Abrahamsen, 2005; Bechtel, 2006; Glennan, 2010 for examples of constitutive mechanisms). Such philosophical work has also explicitly identified critical differences between these kinds of mechanistic explanations (e.g., Levy \& Bechtel, 2016; Kaiser \& Krickel, 2016; Rosenberg, 2020). Consider the questions "How does X work?" vs. "How did X come to exist?" Both are 'how' questions seeking mechanistic explanations, but they are clearly seeking very different kinds of information. In general, etiological explanations require a linear chain of events that has already fully completed, whereas constitutive mechanisms typically involve ongoing processes composed of internal parts (Salmon, 1984; Craver, 2001; Glennan, 2009).

Most research in psychology has been concerned with either etiological or constitutive mechanistic explanations - but without distinguishing between them (e.g., Banerjee \& Bloom, 2014; Kelemen, 1999; Kelemen \& DiYanni, 2009; Lockhart et al., 2019; Schachner et al., 2006, Springer \& Keil, 1989). Might a more complete picture of mechanistic explanation change how we understand and interpret people's explanation preferences? In particular, might people be drawn to one type of mechanism but not the other?

Classifying the kinds of mechanism presented in existing work supports this hypothesis. Work that emphasizes the particular allure of mechanistic explanations (e.g., Buchanan \& Sobel, 
2011; Lockhart et al., 2019; Keil \& Lockhart, in press) tends to present constitutive explanations. Take for example, the following mechanistic explanation of a refrigerator: "The inside pump is able to push lots of the cooling liquid through pipes so the refrigerator always stays cold" (Lockart et al., 2019). This explanation refers to internal pieces (the pump, liquid, and pipes) of the refrigerator, thereby explaining an item by reference to a 'lower level' of constitutive parts and how they work with each other. Some of this research even refers explicitly to constitutive mechanisms (e.g., Lockhart et al., 2019; Keil \& Lockhart, in press) or frames information specifically as "how things work" (Trouche et al., 2018).

In contrast, work that emphasizes the allure of teleological explanations over mechanistic explanations (e.g., Kelemen, 1999; Kelemen \& DiYanni, 2009, Schachner et al., 2006) tends to present etiological mechanistic explanations. Take for example, the following mechanistic explanation of an animal's tail: "The mononykus has a long tail because its feathers were big and stuck out from behind its body" (Kelemen, 1999). This explanation refers to a chain of events that have already been completed (e.g., first the feathers grew large and then they stuck out from the body), and thus seems better characterized as an etiological mechanism.

\section{Current Study}

In this paper, we explore the distinction between etiological and constitutive mechanisms, with two primary questions in mind. First, do people generally distinguish between these types of mechanism, perhaps preferring one over the other? And second, does this distinction capture something meaningful about explanation preferences more broadly, helping to explain the conflicting results on mechanistic vs. teleological explanation preferences?

In Experiments 1 and 2, we evaluate constitutive mechanisms directly against etiological mechanisms. We ask people to choose between them, both by contrasting questions prompting 
etiological vs. constitutive explanations (e.g., how things work vs. how they came to exist;

Experiment 1) and by directly contrasting the explanations (Experiment 2). In Experiments 3, 4a, and $4 \mathrm{~b}$, we examine the performance of etiological and constitutive mechanisms against teleological explanations, again with both questions (Experiment 3) and explanations (Experiments 4a and 4b). We submit that people do recognize the difference between etiological and constitutive mechanisms, and that this difference can in turn help explain differences in people's explanation preferences. We discuss the implications of these findings for the broader questions heavily implicated in the debate on explanation preferences, including the relationship between everyday human cognition and scientific understanding.

\section{Experiment 1: Etiological and Constitutive Questions}

Do people distinguish between etiological and constitutive mechanisms? If so, we might expect that they have specific preferences for one kind of explanation over the other. Here, we ask participants to choose between questions prompting either an etiological or a constitutive mechanistic question. They were then asked which question they would rather have answered: "How does this process work?" (a question requiring a constitutive mechanistic answer) or "How did its circulatory system develop?" (a question requiring an etiological mechanistic answer)."

\section{$\underline{\text { Method }}$}

One hundred adult participants completed a survey online through Amazon Mechanical Turk. The sample size was chosen on the basis of independent pilot data and was preregistered. All participants lived in the United States. Participants were presented with twelve GIFs depicting some object or entity within a dynamic process, four each in the domain of animals,

\footnotetext{
${ }^{*}$ We focus on etiological explanations that describe the development of individual entities from origin to end states, rather than processes that lead to the evolution of a kind of animal or class of designed machines. See also General Discussion.
} 
non-living natural kinds, and artifacts. They were asked to choose between an etiological 'how' question (e.g., "How did x come to be?") and a constitutive 'how' question (e.g., "How does x work?") on the basis of which they would rather have answered. For example, participants were shown an owl twisting its neck 180 degrees, with the caption "This owl has an unusual neck. It can twist its neck almost all the way around in a circle." They were then asked "Which question would you rather have answered?" between "How does this twisting process work?" and "How did its neck come to exist?".

All participants saw all twelve items (in a different random order for each participant), and the order of the questions themselves was also randomized. No other information was collected. For the sake of consistency and comparison with earlier experiments (see also Joo, Yousif, \& Keil, 2020; Kelemen, 1999), data were analyzed in both through simple binomial tests and through t-tests treating participants' scored responses as averages. Redundant analyses are not reported. Data, full materials, and preregistration information for this experiment and all following can be found on the Open Science Framework (OSF) at https://osf.io/e8vzt/?view only=0ee8bbdbf0b04fd2adc1ed92f533c346.

\section{$\underline{\text { Results and Discussion }}$}

To analyze participants' responses, we scored their responses (where a constitutive answer was scored as 1 and an etiological answer as -1) and treated the sum across a domain as an average. Across all domains, people preferred the constitutive question to the etiological question (all $p<.001$ ). These preferences were stronger for animals and artifacts than for NLNKs, all $t(99)>4.0, p<.001$. These results suggest that people distinguish between kinds of mechanistic explanations, as they have systematic preferences to learn about one kind of information over the other. 


\section{Experiment 2: Etiological and Constitutive Explanations}

The previous experiment demonstrated that people prefer to learn about constitutive mechanistic explanations over etiological mechanistic explanations, but do they exhibit this same preference when choosing between the answers to those respective questions? Here, we present participants with an etiological and a constitutive explanation of each of the same twelve phenomena and ask them "Which explanation is more informative?"

\section{$\underline{\text { Method }}$}

All elements of the experimental design were identical to those of Experiment 2, except as stated below. One hundred new participants completed the survey online through Amazon Mechanical Turk. Participants were presented with an etiological and constitutive explanation relating to the item in each GIF asked to choose "Which explanation is more informative?". For instance, for the owl, participants were asked to choose between "The bones in the owl's neck have large openings that their veins and arteries can slide through and not break while twisting" and "As the bones in the neck grew, the openings for the veins and arteries enlarged; then the veins and arteries grew in and through them." Explanations were roughly matched for complexity and length.

\section{$\underline{\text { Results and Discussion }}$}

Across all domains, people preferred the constitutive explanation to the etiological explanation (all $p<.001, d>.80$ ). Unlike in Experiment 1, participants preferred the constitutive mechanism just as much for non-living natural kinds as for animals $(t(198)=.10, p>.05)$. However, they had stronger preferences for both animals and non-living natural kinds than for artifacts, all $t(99)>2.8, p<.02, d>2.8$. 
Note that in this particular experiment, people had stronger preferences for constitutive mechanisms for animals and non-living natural kinds, while artifacts were different. Given, however, the similarity between people's preferences for animals and artifacts (and differences for non-living natural kinds) across our other experiments, these domain-specific preferences seem anomalous to this experiment.

In general, across Experiments 1 and 2, we find that people prefer the constitutive explanations. These results thus suggest that there is a meaningful distinction between these two sub-types of mechanistic explanations. In the following experiments, we explore how this within-mechanism explanation preference impacts people's explanation preferences more broadly. Namely, do people exhibit different preferences regarding teleological explanations when these explanations are paired with either etiological or constitutive mechanisms?

\section{Experiment 3: Mechanistic and Teleological Questions}

Given that people tend to prefer constitutive mechanisms to etiological mechanisms, might constitutive mechanisms fare better against teleological explanations as well? Here, we adapted stimuli from a classic study demonstrating teleological explanation preferences that included etiological mechanisms (e.g., "The mononykus has a long tail because their feathers were big and stuck out from behind their body.”; Kelemen, 1999). 'We again began by investigating people's preferences for different questions. We first obtained people's baseline explanation preferences by contrasting a question demanding etiological mechanistic information (e.g.., "How did the mononykus' tail become long?) with one demanding teleological information (e.g., "What is the purpose of the mononykus' long tail?"); We then contrasted a question demanding constitutive mechanistic information (e.g., "How does the

\footnotetext{
${ }^{\dagger}$ Especially in the case of non-living natural kinds, this systematic generation resulted in some unnatural questions. See Materials and Methods for details.
} 
mononykus keep its balance?") with the same teleological question. In both cases, we asked participants to choose the question that they would rather have answered. Our primary goal was to compare people's mechanistic vs. teleological preferences between these conditions.

\section{$\underline{\text { Method }}$}

All elements of the experimental design were identical to those of previous experiments, except as stated below. Two hundred new participants completed the survey online through Amazon Mechanical Turk. Participants were presented with twelve images, four each of animals, non-living natural kinds, and artifacts.

To establish people's baseline preferences, half of the participants were asked to choose between an etiological 'how' question (e.g., "How did x form?") and a teleological 'purpose' question (e.g., What is the purpose of x?") based on which they would rather have answered. For example, participants were shown an image of a dinosaur with a long tail and asked to choose between "How does the mononykus keep its balance when it runs?" and "What is the purpose of the mononykus' tail?”. These items were adapted from Kelemen, 1999 with the following changes: (1) Participants evaluated questions rather than explanations; (2) All items were shown in a random order; (3) Artifacts were added as a domain; and (4) Extraneous (child-friendly) language was removed for simplicity. Note, these data were originally collected as part of a separate project, and so are also reported in another paper, currently under review (Joo, Yousif, \& Keil, under review).

For our key comparison, the other half of the participants were asked to choose between a constitutive 'how' question (e.g., "How does x do y?") and the same teleological 'purpose' question. The wording of these constitutive 'how' questions was adapted from the original mechanistic explanations presented in Kelemen (1999). Closely adapting existing stimuli 
allowed for a comparison with existing work; however, it also resulted in some cases in unnatural questions (e.g., "How did the rocks stay pointy?”). In general, the existence of constitutive mechanisms in the non-living natural world (especially for simple entities like rocks) is an open philosophical question (see also Keil \& Lockhart, in press). We note that this difficulty may likely be why participants in this experiment were more likely to choose the teleological question when compared to an etiological vs. constitutive mechanism for non-living natural kinds, even though participants in Experiment 1 preferred constitutive mechanisms over etiological mechanisms.

\section{$\underline{\text { Results and Discussion }}$}

The key analysis of this experiment compares people's preferences in this task to their preferences when evaluating identical 'purpose' questions against etiological 'how' questions (e.g., "How did x come to be?"). This comparison was explicitly preregistered and the two experiments were run at similar times.

We found that people's preferences between a constitutive mechanistic question and a teleological question were clearly different from their preferences between an etiological mechanistic question and a teleological question. When comparing etiological and teleological questions, people tended to prefer 'purpose' questions when asked about animals, $(M=3.01, S D$ $=1.12, t(99)=8.99, p<.001, d=.90)$ and artifacts $(M=3.68, S D=.65, t(99)=25.87, p<.001$, $d=2.59)$ — but etiological 'how' questions for NLNK, $(M=.80, S D=.99, t(99)=12.19, p<.001, d$ $=1.22$ ). In contrast, participants ubiquitously preferred 'purpose' questions when thsee were contrasted with constitutive mechanistic questions, across animals, $(M=2.58, S D=1.02$, $t(99)=5.70, p<.001, d=.57)$; NLNK, $(M=2.24, S D=.99, t(99)=2.43, p=.02, d=.24)$; and artifacts, $(M=2.49, S D=.89), t(99)=5.49, p<.001, d=.55)$. In most cases, therefore, people judged 
constitutive questions (as opposed to etiological questions) more favorably against teleological questions; this was true for both animals, $t(198)=2.84, p=.005, d=.40$, and artifacts, $t(198)=10.78$, $p<.001, d=1.52$. For non-living natural kinds, (a domain where people already have strong preferences for etiological over teleological information) participants were less inclined to choose the constitutive mechanistic questions, $t(198)=10.33, p<.001, d=1.46$. In general, the extent to which people seek mechanism is dependent on the kind of mechanism being offered. Still, people sought teleological information to a moderate extent. In other words, these results don't suggest that people never prefer teleology, but that their preferences meaningfully shift depending on the contrasting mechanism.

\section{Experiments 4a and 4b: Mechanistic and Teleological Explanations}

In the previous experiment, we compared the performance of constitutive or etiological mechanisms against teleological information by comparing participants' preferences for questions prompting each of these kinds of information. Mimicking the design of Experiment 2, here we asked about explanation preferences directly. We again began by examining people's baseline explanation preferences by contrasting teleological explanations with etiological explanations (e.g., "The mononykus has a long tail because its feathers were big and stuck out from behind its body"). We adapted these explanations from Kelemen (1999) with minimal changes, and replicated people's known explanation preferences.

In Experiment 4a, we sought to test the impact of constitutive vs. etiological explanations by closely continuing to closely adapt from existing work (as in Experiment 3 ). Here, we contrasted teleological explanations with constitutive explanations (e.g., "The mononykus swings its tail when it runs because its tail is attached by a kind of joint that slides in and out"). 
Participants were asked to choose an explanation in response to a 'why' question, on the basis of "which explanation makes the most sense?".

In order to address the potentially unnatural explanations created by the systematic generation from existing stimuli, in Experiment $4 \mathrm{~b}$ we compared the same etiological vs. teleological contrast against original stimuli contrasting constitutive vs teleological explanations. These stimuli were all dynamic processes that were presented with gifs. Participants were again asked to choose with explanation made the most sense. In both Experiments $4 \mathrm{a}$ and $4 \mathrm{~b}$, our primary goal was to compare people's explanatory preferences between conditions of different kinds of mechanisms - in other words, to compare their preferences between constitutive mechanistic vs. teleological explanation preferences against their baseline preferences between etiological vs. teleological explanations.

\section{$\underline{\text { Method }}$}

All elements of the experimental design were identical to Experiment 3, except as stated below. Three hundred new participants completed the survey online through Amazon Mechanical Turk. All participants were shown the same twelve items (four animals, non-living natural kinds, and artifacts) as in Experiment 3.

To establish people's baseline preferences, the first group of one hundred participants were asked to evaluate a teleological vs. an etiological mechanistic explanation (in response to a 'why' question) on the basis of which explanation made the most sense. For example, participants were asked "Why does the mononykus have such a long tail?" and asked to choose between "The mononykus has a long tail because its feathers were big and stuck out from behind its body" and "The mononykus has a long tail so that it can keep its balance when it runs". These explanations were adapted from Kelemen (1999) with minimal changes. Note, these data were 
originally collected as part of a separate project, and so are also reported in another paper, currently under review (Joo, Yousif, \& Keil, under review).

For our key contrast in Experiment 4a, one hundred participants were asked to evaluate a teleological vs. a constitutive mechanistic explanation, again with stimuli closely adapted from Kelemen (1999). For instance, participants were asked "Why does the mononykus swing its tail when it runs?" and asked to choose between "The mononykus swings its tails when it runs because its tail is attached by a kind of joint that slides in and out" and "The mononykus swings its tail when it runs so that it can keep its balance." Teleological explanations were adapted nearverbatim from Kelemen, 1999 (changed only to avoid redundancy with the 'why' question that both explanations addressed). In order to present constitutive, rather than etiological, mechanistic explanations, these 'why' questions asked about dynamic processes rather than static features. Each constitutive mechanistic explanation also described the interaction of internal parts, thus meeting the two major criteria of constitutive explanations. As in Experiment 3, however, this systematic derivation of constitutive explanations led to some explanations which were either unnatural or not very constitutive (see discussion of Experiment 3), e.g., "The rocks aren't sat on because their pointy tops poke any animals that try."

For our key contrast in Experiment 4b, one hundred participants were presented with a contrast between constitutive and teleological explanations — but with original stimuli. Here, we intentionally used stimuli that were not modeled on prior work in an attempt to provide plausible questions and explanations in all domains. Participants were presented with six GIFs of dynamic processes (note: not the same items as used in Experiments 1-2), two each of animals, non-living natural kinds, and artifacts. These items were chosen to present actual dynamic processes (as opposed to attempts to engineer them from existing stimuli in Experiments 3 and 4a) while being 
as similar as possible to previous stimuli. Participants, for instance, were shown a swimming dinosaur and asked "Why is the cryptoclidus swimming in a wave motion?", choosing between "It's swimming in a wave motion because little pieces in its neck and back move one by one" and "It's swimming in a wave motion so that it can move without splashing and sneak up on its food."

The key question in both experiments $4 \mathrm{a}$ and $4 \mathrm{~b}$ was again a comparison between people's explanation preferences when comparing an etiological vs. a teleological explanation as opposed to a constitutive vs. a teleological explanation, and this comparison was explicitly preregistered.

\section{$\underline{\text { Results and Discussion }}$}

The key question in both experiments $4 \mathrm{a}$ and $4 \mathrm{~b}$ was again a comparison between people's explanation preferences when comparing an etiological vs. a teleological explanation as opposed to a constitutive vs. a teleological explanation, and this comparison was explicitly preregistered.

When etiological and teleological explanations were contrasted, people preferred the teleological explanations for animals, $(M=3.55, S D=.82, t(99)=18.88, p<.001, d=1.88)$ and artifacts, $(M=3.48, S D=.80, t(99)=18.56, p<.001, d=1.86)$-but the etiological mechanistic explanations for non-living natural kinds, $(M=.38, S D=.76, t(99)=21.25, p<.001, d=2.13)$. These results replicate known explanation preferences. In Experiment 4a, participants still tended to prefer teleological explanations about animals, $(M=3.19, S D=.92, t(99)=12.97, p<.001, d=1.30)$, and mechanistic explanations about NLNK, $(M=.08, S D=.31, t(99)=62.44, p<.001, d=6.24)$. However, their explanatory preferences about artifacts were not significantly mechanistic or teleological, $(M=2.19, \mathrm{SD}=1.11, t(99)=1.72, p=.09)$. Critically, participants' were more likely in 
all domains to prefer a mechanistic over a teleological explanation when presented with a constitutive vs. etiological mechanism, all $t(198)>2.9, p<.005, d>$.4. In some cases these shifts are small (e.g., for the animals, 89\% of participants preferred a teleological explanation over an etiological explanation, whereas only $80 \%$ preferred the teleological explanation over a constitutive explanation), but others are quite dramatic (e.g., for artifacts, $87 \%$ of participants preferred a teleological explanation over an etiological explanation, whereas only $55 \%$ did with constitutive explanations). In all cases, these subtle shifts are important, as they suggest that people's preferences are indeed sensitive to differences in mechanistic explanatory kind.

In Experiment 4b, with perhaps a fairer comparison between constitutive and teleological explanations, participants now preferred teleological explanations about animals, $(M=1.29$, $S D=.72, t(99)=4.06, p<.001, d=.41)$; but mechanistic explanations about NLNK, $(M=.52$, $S D=.63, t(99)=7.65, p<.001, d=.77)$, and artifacts, $(M=.21, S D=.48, t(99)=16.54, p<.001$, $d=1.65)$. In contrast with their preferences between etiological and teleological explanations, participants' preferences were even more clearly shifted towards mechanism than in Experiment 4a in the domains of animals and artifacts, $t(198)=5.88, p<.001, d=.83)$ and artifacts, $t(198)=24.59, p<.001, d=3.48$. (For non-living-natural kinds, this pattern reversed, $t(198)=4.50$, $p<.001, d=.64-$ but we note that people's preferences in this domain for etiological explanations over teleological explanations are already near ceiling, and that their preferences in this experiment seemed driven by a single item.)

\section{General Discussion}

Across five experiments, we find not only that people are sensitive to the differences between etiological and constitutive mechanisms, but that this distinction has clear consequences for explanation preferences more broadly. In general, people prefer constitutive mechanisms to 
etiological mechanisms, both when asked to evaluate questions (Experiment 1) and when asked to evaluate explanations (Experiment 2). Further, these within-mechanism preferences reshape how we think about other known explanation preferences: The extent to which people preferred mechanism was dependent on the kind of mechanism offered (i.e., people are more likely to seek/prefer mechanism when that mechanism is constitutive rather than etiological; Experiments $3,4 a$, and $4 b)$.

In short: (1) there are two sub-types of mechanistic explanations (constitutive vs. etiological); (2) people generally prefer constitutive over etiological explanations; (3) prior work on explanation preferences has not taken into account these sub-types of mechanistic explanation; and (4) if these sub-types are taken into account, people no longer have global teleological or mechanistic preferences. Collectively, this pattern of results suggests a different view of human explanation preferences, one that is flexible and context-specific.

\section{A taxonomy of mechanistic explanations?}

The category 'mechanistic explanations' requires at least two subdivisions between etiological and constitutive mechanisms. This division is demonstrated not only by people's clear preferences for constitutive over etiological mechanisms, but also by the fact that people consider these explanations differently in contrast with teleological explanations. Future work may want to examine how these particular types of mechanism vary across other existing experiments. For instance, might someone who knows constitutive mechanistic information be considered more of an expert than someone who knows etiological mechanistic information (c.f. Lockhart et al., 2019)?

Our work breaks down mechanism into two subcategories of explanation, ones that are sufficiently distinct so as to affect people's broad explanation preferences. In principle, however, 
the taxonomy of mechanistic explanations may be even more detailed (and future work may be interested in a more precise relationship between various subtypes). For instance, parts of animals seem to have two possible types of etiological explanations: one which describes the development of a feature from embryo to adulted and another which describes its evolution over time. That said, we think that the larger distinction between etiological and constitutive mechanisms may be sufficient to explain most of the variability in explanation preferences across existing studies.

\section{Mech-centric vs. teleo-centric explanation preferences?}

The sub-types of mechanistic explanation have a clear impact on people's explanation preferences, demonstrating a need for the larger debate on explanation preferences (and their consequences for theories of human cognition) to take this distinction into account. Do people seek causally rich, mechanistic information, or do they prefer teleological explanations in a broad array of contexts? The answer may be both-but perhaps not to the extent that either these views have proposed.

In some cases, people prefer constitutive mechanisms - not only in contrast to etiological mechanisms, but also in contrast to teleology. We demonstrate that constitutive mechanistic explanations are more appealing in contrast to teleological explanations than their etiological counterparts (Experiments $4 \mathrm{a}$ and $4 \mathrm{~b}$ ). These results can contextualize why existing research supporting the mech-centric view of explanation often presents constitutive explanations: for instance, directly asking for "how things work" (e.g., Grief et al., 2006; Trouche et al., 2018) or providing explanations about internal parts interacting in an ongoing process (definitional to constitutive mechanisms; e.g., Buchanan \& Sobel, 2011; Chuey et al., 2020; Lockhart et al., 2019). Might constitutive mechanisms, in particular, be alluring explanations? These 
explanations are sometimes identified as uniquely useful in science, especially in biology, because of their ability to explain a phenomenon in ever-increasing depth (Bechtel, 2011; Craver \& Darden, 2013). Take, for instance, understanding how the heart pumps blood: This constitutive explanation might refer to how arteries, veins, valves, and chambers interact to pump blood-yet each of these component parts also have constitutive explanations, which might refer to muscle fibers, blood vessels, or internal valves. Perhaps even in everyday contexts, constitutive mechanistic explanations are able to convey a unique depth of understanding because of this potential to richly unpack underlying details. The appeal of constitutive mechanistic explanations suggests that people are interested in not only causal explanations (in which case they would also prefer etiological explanations), but in these more complex causal relationships.

In other cases, however, people prefer teleology, including in contrast with (etiological) mechanisms. The linear chain of events presented in an etiological mechanism may not be as richly layered, but is nevertheless a kind of causal explanation. People's preferences for teleology over etiological mechanisms (e.g., Banerjee \& Bloom, 2015; Kelemen, 1999; Kelemen \& DiYanni, 2009) does therefore demonstrate that people do sometimes prefer or endorse explanations that fail to provide causal information — such as when adults and children endorse teleological explanations about non-living natural kinds (e.g., Heywood \& Bering, 2014; Kelemen et al., 2013; Kelemen \& Rosset, 2009). Our results indicate not that people never prefer teleology, but that these preferences are not as absolute as previously characterized. In some cases, teleology may simply have been contrasted with a less-alluring type of mechanism. Moreover, the flexibility of people's preferences may arise from interactions between these types of explanation. To fully explain how a complex machine works, for instance, may 
require reference to the functions of individual components. In cases like these, constitutive mechanism and function work together (see also Craver, 2001; Cummins, 1975). In other cases, etiological mechanism and function may also complement each other. Consider evolution by natural section: The function of something like a giraffe's long neck is part of explaining how particular adaptations became prevalent (even without intentional teleological explanations; see also Garson, 2012). In reality, teleology and both kinds of mechanism may often work hand in hand - and people's particular preferences may reflect the subtle value of one kind of information over another in a specific context.

\section{Broader Implications}

The experiments reported here offer a way to understand how both the teleo-centric and mech-centric views present co-existing truths about people's explanation preferences. Yet both views also offer conflicting perspectives on how to best characterize human cognition: While the mech-centric view argues that people are fundamentally curious about the rich causal structure of the world (e.g., Callanan \& Oakes, 1992; Greif et al., 2006; Trouche et al., 2018), some versions of the teleo-centric view go so far as to say that human cognition is fundamentally unscientific (e.g., Kelemen, 1999; Kelemen et al., 2013; Rose \& Schaffer, 2017). Our results suggest a more nuanced understanding of people's explanation preferences: People prefer different, specific kinds of information, rather than having a default preference for teleology or mechanism.

People's explanation preferences may therefore be sensibly dependent on informational contexts, and their teleological preferences may simply be indicative of cases where the teleological explanation provided more useful or interesting information. In other words, when the mechanistic explanation is of a compelling or richly structured kind, people are-quite reasonably — more likely to prefer these explanations. Take, for instance, the example of 
encountering an everyday machine. It may be that knowing how something like a microwave works is richly informative, but that knowing how it was assembled is less so. In contrast to this latter etiological explanation, a teleological explanation may provide a more compelling alternative. Here, the purpose of the microwave can provide useful information on how to use the microwave and allows for rich inferences about when and how other people are likely to interact with it (see also Joo, Yousif, \& Knobe, 2020).

This level of sensitivity to subtypes of explanation requires reevaluation of how to broadly make sense of people's explanation preferences. If people preferred teleology ubiquitously, without consideration of the particular information being imparted, then we might be more likely to think their default is a mistaken view of causal explanation. Instead, however, we find that teleology preferences are not as promiscuous as previously thought. At the same time, it's not the case that people always prefer mechanism. There are meaningful subtypes that they prefer - and even constitutive mechanisms are not ubiquitously favored over teleological explanations. Similarly, explanation preferences may indicate neither that human cognition is inherently unscientific nor that it is always attuned to causal learning.

\section{Conclusion}

Both as scientists and throughout our everyday lives, we seek to understand and to explain the world around us. The appeal of different kinds of explanations and the consequences of these preferences have long been topics of interest for cognitive scientists. Over the last few decades, the mech-centric and teleo-centric views have emerged as major, contrasting ways of understanding human explanation (as well as human cognition). Here, however, we suggest that a distinction between etiological and constitutive mechanistic explanations may help us to 
reevaluate both views, providing a critical step towards reconciling this debate as we move forward. 


\begin{abstract}
Author Note
For helpful comments and conversation we thank Joshua Knobe, Aaron Chuey, an anonymous reviewer, and all members of the Yale Cognition and Development Lab. This project was supported by a National Science Foundation Graduate Research Fellowship awarded to S. R. Yousif. Data, full materials, and preregistration information for this experiment and all following can be found on the Open Science Framework (OSF) at https://osf.io/e8vzt/?view only=0ee8bbdbf0b04fd2adc1ed92f533c346.
\end{abstract}

The authors have no known conflicts of interest to report. 


\section{References}

Banerjee, K., \& Bloom, P. (2015). “Everything Happens for a Reason”: Children's Beliefs About Purpose in Life Events. Child Development, 86, 503-518.

Barros, D.B. (2008). Natural Selection as a Mechanism. Philosophy of Science, 75, 306-322.

Bechtel, W. (2006). Discovering Cell Mechanisms: The Creation of Modern Cell Biology. Cambridge: Cambridge University Press.

Bechtel, W. (2011). Mechanism and Biological Explanation. Philosophy of Science, 78, 533-557.

Bechtel, W. \& Abrahamsen, A. (2005). Explanation: A Mechanistic Alternative. Studies in History and Philosophy of the Biological and Biomedical Sciences, 36, 421-441.

Buchanan, D. W., \& Sobel, D. M. (2011). Mechanism-based causal reasoning in young children. Child Development, 82, 2053-2066.

Callanan, M. A., \& Oakes, L. M. (1992). Preschoolers' questions and parents' explanations: Causal thinking in everyday activity. Cognitive Development, 7, 213-233.

Chouinard, M. M., Harris, P. L., \& Maratsos, M. P. (2007). Children's questions: A mechanism for cognitive development. Monographs of the Society for Research in Child Development, i-129.

Chuey, A., Lockhart, K., Sheskin, M., \& Keil., F.C. (2020). Children and adults selectively generalize mechanistic knowledge. Cognition, 199, 104231.

Corriveau, K. H., \& Kurkul, K. E. (2014). “Why does rain fall?”: Children prefer to learn from an informant who uses noncircular explanations. Child Development, 85, 1827-1835.

Craver, C.F. (2001). Role Functions, Mechanisms and Hierarchy. Philosophy of Science, 68, 3155. 
Craver, C.F. \& Darden, C.F. (2013). In Search of Mechanisms: Discoveries Across the Life Sciences. Chicago: University of Chicago Press.

Cummins, R. (1975). Functional Analysis/ Journal of Philosophy, 72, 741-764.

Engel, S. (2011). Children's need to know: Curiosity in schools. Harvard Educational Review, $81,625-645$.

Erickson, J. E., Keil, F. C., \& Lockhart, K. L. (2010). Sensing the coherence of biology in contrast to psychology: Young children's use of causal relations to distinguish two foundational domains. Child Development, 81, 390-409.

Frazier, B. N., Gelman, S. A., \& Wellman, H. M. (2009). Preschoolers' search for explanatory information within adult-child conversation. Child Development, 80, 1592-1611.

Frazier, B. N., Gelman, S. A., \& Wellman, H. M. (2016). Young children prefer and re- member satisfying explanations. Journal of Cognition and Development, 17, 718-736.

Garson, J. (2011). Selected Effects Functions and Causal Role Functions in the Brain: The Case for an Etiological Approach to Neuroscience. Biology \& Philosophy, 26: 547-565.

Gelman, S. A., \& Wellman, H. M. (1991). Insides and essences: Early understandings of the non-obvious. Cognition, 38, 213-244.

Glennan, S. S. (2009). Productivity, Relevance and Natural Selection. Biology and Philosophy, 24, 325-339.

Glennan, S. (2010). Ephemeral Mechanisms and Historical Explanation. Erkenntnis, 72, 251-66.

Greif, M. L., Nelson, D. G. K., Keil, F. C., \& Gutierrez, F. (2006). What Do Children Want to Know About Animals and Artifacts? Psychological Science, 17, 455-459. 
Heywood, B. T., \& Bering, J. M. (2014). "Meant to be": How religious beliefs and cultural religiosity affect the implicit bias to think teleologically. Religion, Brain \& Behavior, 4, 183-201.

Joo, S., \& Yousif, S. R. (2021, January 7). Understanding “How.” Retrieved from osf.io/e8vzt

Joo, S., Yousif, S. R., Keil, F. C. (2020). Implicit questions shape information preferences. Proceedings of the $42^{\text {nd }}$ Annual Conference of the Cognitive Science Society. Toronto, ON: Cognitive Science Society.

Joo, S., Yousif, S. R., Keil, F. C. (under review). Understanding 'Why': How implicit questions shape information preferences. under

Joo, S., Yousif, S., Knobe, J. (2020). Teleology beyond explanation: What factors influence teleology judgments? PsyArXiv Preprints.

Kaiser, M. I. \& Krickel, B. (2017). The Metaphysics of Constitutive Mechanistic Phenomena. The British Journal for the Philosophy of Science, 68, 745-779.

Keil, F. C. \& Lockhart, K. L. (In press). Beyond Cause: The Development of Clockwork Cognition. Current Directions in Psychological Science.

Keil, F. C., Lockhart, K. L., \& Schlegel, E. (2010). A bump on a bump? Emerging intuitions concerning the relative difficulty of the sciences. Journal of Experimental Psychology: General, 139, 1.

Keil, F. C., Stein, C., Webb, L., Billings, V. D., \& Rozenblit, L. (2008). Discerning the division of cognitive labor: An emerging understanding of how knowledge is clustered in other minds. Cognitive Science, 32, 259-300.

Kelemen, D. (1999). Why are rocks pointy? Children's preference for teleological explanations of the natural world. Developmental Psychology, 35, 1440-1452. 
Kelemen, D. (2004). Are Children "Intuitive Theists"?. Reasoning About Purpose and Design in Nature. Psychological Science, 15, 295-301.

Kelemen, D., \& Diyanni, C. (2005). Intuitions About Origins: Purpose and Intelligent Design in Children's Reasoning About Nature. Journal of Cognition and Development, 6, 3-31.

Kelemen, D., \& Rosset, E. (2009). The Human Function Compunction: Teleological Explanation in Adults. Cognition, 111, 138-143.

Kelemen, D., Rottman, J., \& Seston, R. (2013). Professional Physical Scientists Display Tenacious Teleological Tendencies: Purpose-Based Reasoning as a Cognitive Default. Journal of Experimental Psychology: General, 142, 1074-1083.

Lockhart, K. L., Chuey, A., Kerr, S., \& Keil, F. C. (2019). The privileged status of knowing mechanistic information: An early epistemic bias. Child Development, 90, 1772-1788.

Levy, A., \& Bechtel, W. (2016). Towards Mechanism 2.0. Expanding the Scope of Mechanistic Explanation. In PSA 2016. The 25th Biennial Meeting of the Philosophy of Science Association.

Lewry, C., Lombrozo, T., \& Kelemen, D. (2020). People view humans as existing for purposes and condemn those who fail to fulfill them. Proceedings of the $41^{\text {st }}$ Annual Meeting of the Cognitive Science Society. Toronto: ON: Cognitive Science Society.

Rose, D. (Forthcoming). Mentalizing Objects. In Oxford Studies in Experimental Philosophy 4. Oxford, England: Oxford University Press.

Rose, D. (2015). Persistence through function preservation. Synthese, 192, 97-146.

Rose, D., \& Schaffer, J. (2017). Folk Mereology is Teleological. Nô̂s, 51, 238-270.

Rose, D., Schaffer, J., \& Tobia, K. (2020). Folk teleology drives persistence judgments. Synthese. 
Rosenberg, A. (2020). Reduction and Mechanism (60-66). Cambridge University Press.

Rozenblit, L., \& Keil, F.C. (2002). The Misunderstood Limits of Folk Science: An Illusion of Explanatory Depth. Cognitive Science, 26, 521-562.

Salmon, W. (1984). Scientific Explanation: Three Basic Conceptions. Proceedings of the Biennial Meeting of the Philosophy of Science Association, 293-305.

Schachner, A., Zhu, L., Li, J., \& Kelemen, D. (2017). Is the bias for function-based explanations culturally universal? Children from China endorse teleological explanations of natural phenomena. Journal of Experimental Child Psychology, 157, 29-48.

Skipper Jr., R.A. \& R.L. Millstein. (2005). Thinking about Evolutionary Mechanisms: Natural Selection. In C.F. Craver \& L. Darden (Ed.), In Search of Mechanisms: Discoveries Across the Life Sciences (327-347). Chicago: University of Chicago Press.

Springer, K., \& Keil, F. C. (1989). On the development of biologically specific beliefs: The case of inheritance. Child development, 637-648.

Trouche, E., Chuey, A, Lockhart, K., \& Keil, F.C. (2018). Children Don't Just Wanna Have Fun: An Experimental Demonstration of Children's Curiosity for How Things Work. Proceedings of the $40^{\text {th }}$ Annual Meeting of the Cognitive Science Society. Madison, Wisconsin: Cognitive Science Society. 
Figures and Figure Captions

\section{Experiment 1}

Etiological vs. Constitutive Questions

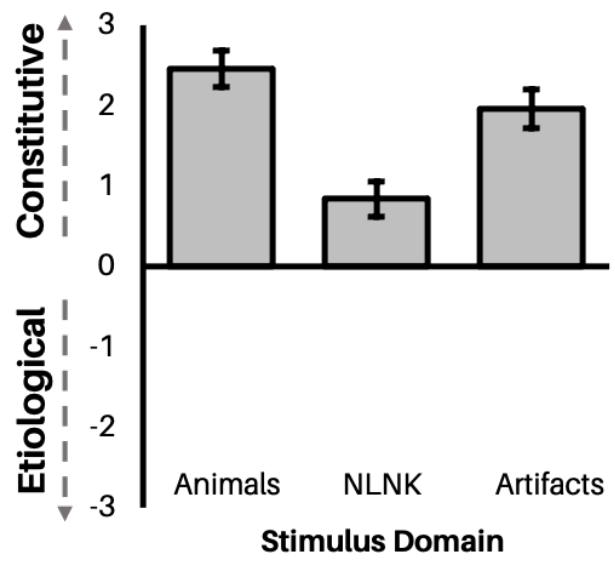

Experiment 2

Etiological vs. Constitutive Explanations

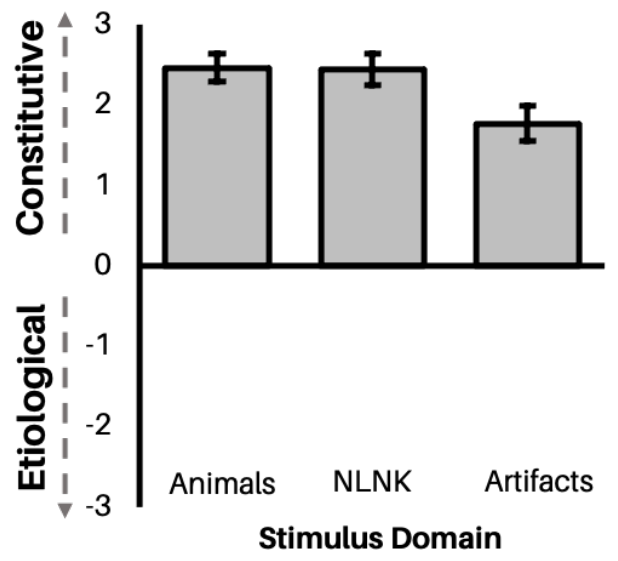

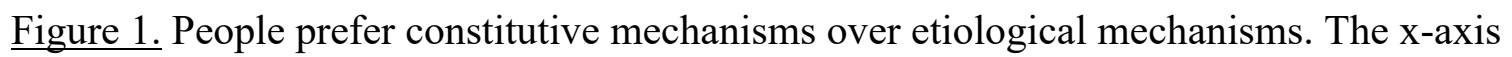
represents chance performance. Domain of the stimulus is represented along the axis.

Participants' scored responses are represented along the y-axis. Error bars represent +/- $1 \mathrm{SE}$. 


\section{Etiological vs. Teleo Questions}

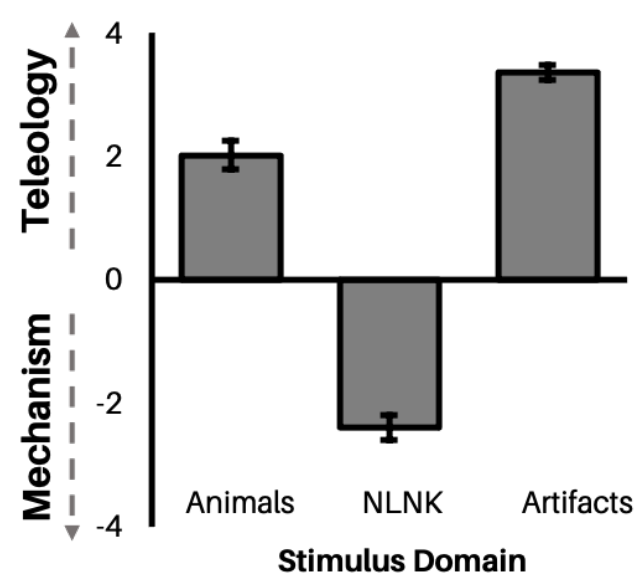

\section{Experiment 3}

Constitutive vs. Teleo Questions

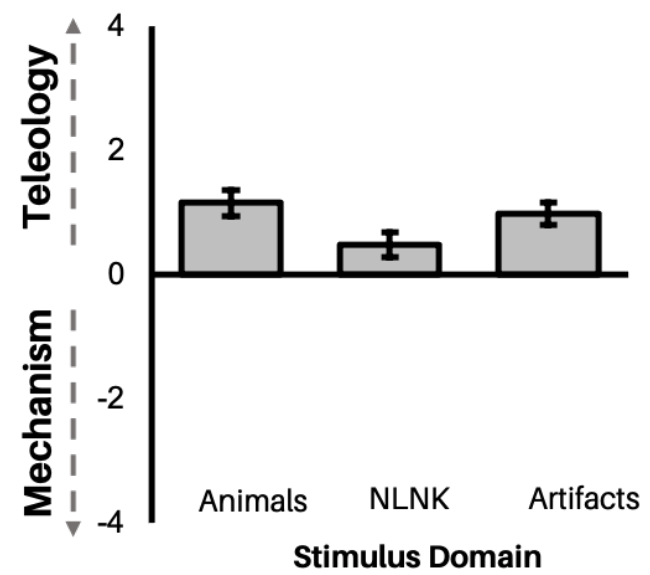

Figure 2. When participants compare constitutive (rather than etiological) mechanistic questions against teleological questions, previously documented teleology preferences are diminished. The $\mathrm{X}$-axis represents chance performance. Domain of the stimulus is represented along the axis. Participants' scored responses are represented along the y-axis. Error bars represent +/- $1 \mathrm{SE}$. 
Etiological vs. Teleo Explanations

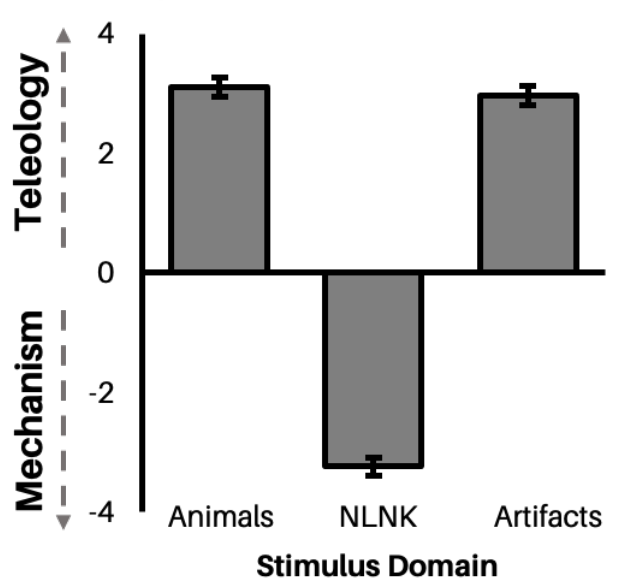

Experiment 4a

Constitutive vs. Teleo Explanations

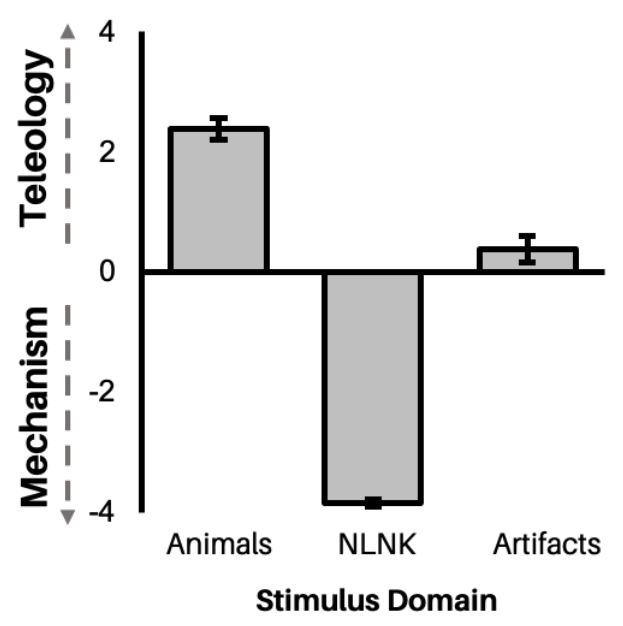

Experiment 4b

Constitutive vs. Teleo Explanations

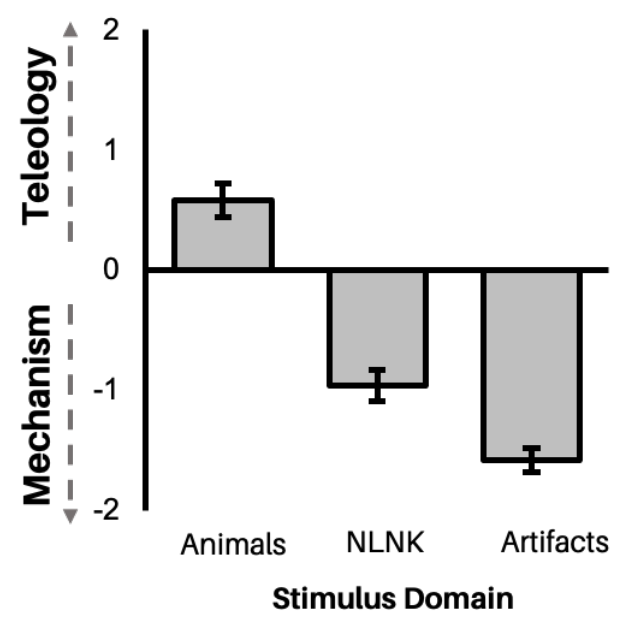

Figure 3. When participants compare constitutive (rather than etiological) mechanistic questions against teleological explanations, previously documented teleology preferences are diminished. The $\mathrm{x}$-axis represents chance performance. Domain of the stimulus is represented along the axis. Participants' scored responses are represented along the y-axis. The axis for the graph of Experiment $4 \mathrm{~b}$ ranges from -2 to 2 (rather than -4 to 4 ) because there were half as many items. Error bars represent +/- $1 \mathrm{SE}$. 